\title{
Heparan sulfate: growth control with a restricted sequence menu
}

\author{
John T. Gallagher \\ Department of Medical Oncology, University of Manchester, Paterson Institute for Cancer Research, Christie Hospital NHS Trust, Wilmslow \\ Road, Manchester, M20 4BX, United Kingdom. Phone: 44-161-446-3201; Fax: 44-161-446-3269; E-mail: JGallagher@picr.man.ac.uk.
}

J. Clin. Invest. 108:357-361. DOI:10.1172/JCI200113713.

The growth and differentiation of cells and their organization into organs and tissues are complex processes, largely determined by the concerted actions of the ECM and small, soluble "effector" proteins in the pericellular milieu. Cells respond to the instructive potential of their microenvironment by means of specialized plasma membrane receptors, which elicit intracellular signals when activated by cognate ECM or soluble peptide ligands. In many receptor systems, ligand binds first to an abundant, low-affinity receptor, which draws the ligand onto the cell surface and then transfers it to a second, high-affinity receptor that transduces the appropriate signal into the cell. The most common and widely acting low-affinity receptors are the heparan sulfate proteoglycans (HSPGs), which play central roles in the reception and modulation of a wide range of growth factors, morphogens, and chemokines (1-3). They also act in combination with membrane integrins to control cell adhesion and migration in the ECM (4).

The question arises as to how the structure and properties of the HSPGs enable them to fulfill such diverse and fundamental roles. These issues are the subject of intense experimental study, and a growing body of data has been published on the protein recognition properties of HSPGs and their association with signaling receptor complexes. However, more questions are being raised than problems solved by these analyses, perhaps as a result of the unusual properties of the HS chains that determine the majority of the interactions of PGs with external proteins. HS polysaccharides display a versatility in conformation and orientation of functional groups that permits them to employ different modes of binding with any individual protein or protein complex. Here, I argue that this versatility could offset the need for the controlled synthesis of a vast repertoire of defined sequences for exclusive binding of individual proteins.

\section{Molecular structure and biosynthesis of heparan sulfate}

HS is synthesized on a restricted group of core proteins associated with the cell surface (the syndecan and glypican families, and an isoform of CD44) or the ECM (perlecan, agrin, and collagen XVIII) (refs. 5, 6; see also Iozzo's introduction to this Perspective series, ref. 7).
The HS chains are polymerized on tetrasaccharideprimed serine residues located in sequence motifs of short Ser-Gly repeats flanked by hydrophobic and acidic domains that favor the synthesis of HS rather than chondroitin sulfate (8). Normally two to three HS chains are found in close proximity along the PG core proteins, suggesting that they act in a concerted manner in controlling cell behavior.

HS is a glycosaminoglycan (GAG) in which the disaccharide repeating unit is composed of $\mathrm{N}$-substituted glucosamine and hexuronic acid. The chain is initially synthesized as an N-acetylated polymer (GlcNAc-GlcA repeat units), which then undergoes extensive but localized modifications, which transform the polymeric "heparan" precursor to HS. This process is described in detail in this Perspective series by Esko and Lindahl (9). In the mature HS chain, about $40-50 \%$ of the amino sugars are converted from GlcNAc to $\mathrm{GlcNSO}_{3}$. The $\mathrm{N}$-sulfated residues occur predominantly in contiguous sequences or S-domains (two-and-seven-eighths disaccharide units in length) with a minor but significant

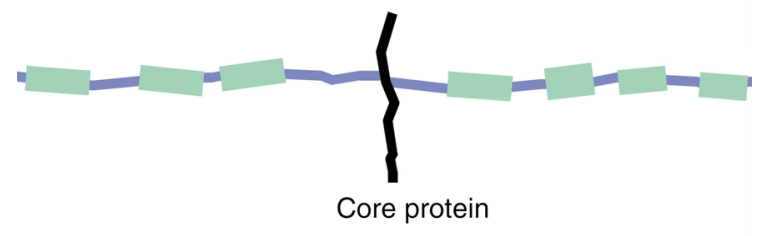

Sulfated (S) domain $\quad$ Flexible spacers

\section{Figure 1}

The sulfated domains of HS are the main regions involved in the recognition of growth factors and other proteins. They are separated by flexible spacers of low sulfation. Although there is a vast potential for sequence diversity in the $\mathrm{S}$-domains, the variation in any one cell type may be restricted as a result of the substrate specificities of the polymer-modifying enzymes that convert the precursor heparan (GlcA-GlcNAc) ${ }_{n}$ to HS. Several proteins will bind to any individual S-domain, often by recognizing different structural features of the domain. Minimal sequences designed for binding exclusively to one protein are predicted to be rare but of major importance in cell biology. Evidence from animals deficient in HS-biosynthetic enzymes suggests that weak GAG-protein interactions may be more significant than was previously realized, and this has implications for understanding the mode of action of HS and for the design of HS/heparin mimetics. 
proportion in alternate or mixed sequences with the original $\mathrm{N}$-acetylated disaccharides. The S-domains are normally distributed in a fairly uniform manner in HS, separated by about 15 disaccharides which are largely made up of unmodified N-acetyl-rich regions (10) (Figure 1); the mixed sequences define transition zones between the S-domains and the unmodified segments. This basic molecular design of spatially discrete sulfated domains is a unique characteristic of HS and may confer distinctive physical and chemical properties on the glycan chain. The one known exception to this arrangement is found in HS from the rat liver, in which the S-domains are located in close proximity in the distal portion of the polysaccharide (11).

The S-domains are the major focus for the additional polymer modifications of C- 5 epimerization of glucuronate to iduronate (IdoA) and O-sulfation at C- 2 of IdoA and C- 6 of GlcNSO$_{3}$; C- 6 sulfation also occurs on the amino sugars in the mixed sequences (12). Most of the uronate residues in the S-domains are converted to IdoA,2S, and therefore the disulfated disaccharide IdoA,2S-GlcNSO 3 is a near-repetitive feature of these regions (13). The degree of 6-O-sulfation of the $\mathrm{S}$-domains and the mixed sequences is variable, and $\mathrm{S}$-domains devoid of 6-O-sulfate groups are relatively common (12-14).

The rare polymer modifications of O-sulfation at C-3 of $\mathrm{GlcNSO}_{3}$ and the deacetylation of GlcNAc to yield an $\mathrm{N}$-unsubstituted glucosamine also occur in the S-domain and mixed sequence regions of HS (15, 16). The low frequency of these modifications belies their potential significance in creating highly specific protein-binding sequences.

\section{Physical properties of heparan sulfate: a flexible polymer}

There have been few analyses of the physical properties of HS, but the chemically related heparin has been thoroughly studied. Heparin is an analogue of the S-domains of HS, and it consists mainly of sequences of trisulfated disaccharides of structure IdoA,2S-Glc$\mathrm{NSO}_{3}, 6 \mathrm{~S}$. Heparin adopts the conformation of a relatively stiff helix, whose helical rotation places clusters of sulfate groups at regular intervals of about $17 \AA ̊$ on either side of the helical axis (17), a distance that approximates the spacing between groups of positively charged amino acid residues in several heparin-binding proteins. This bilateral arrangement of sulfate clusters creates a two-sided polymer that can bind growth factors in trans-binary complexes with enhanced biological activity. In all probability, the S-domains in HS will form a similar helical structure to heparin but with a lower and more variable level of sulfation.

The IdoA residues in heparin (and presumably also in HS) exhibit a remarkable conformational plasticity in comparison with other pyranose rings, oscillating between ${ }^{1} \mathrm{C}_{4}$ and ${ }^{2} \mathrm{~S}_{\mathrm{o}}$ skew boat forms with minimal change in linkage geometries to adjacent sugars (18). The importance of the IdoA ring plasticity in molecular recognition is revealed by $\mathrm{x}$-ray crystallography of a heparin hexasaccharide complexed with FGF2 in which the two internal IdoA residues are locked in different conformations that increase the area of contact with the protein surface (19). Although in solution the heparin helix is somewhat rigid, careful examination of crystal structures of several heparin-protein complexes has revealed a marked distortion or "kink" in the helical axis that further improves the fit with the topological features of the GAG-binding regions of the proteins $(20,21)$.

There is a dearth of information on the physical structure of the GlcA-GlcNAc sequences that form the low sulfated regions of HS, but by comparing data from suitable model compounds, Mulloy and Forster (18) suggest that flexible linkage geometries may characterize these sections of the polymer. This would enable the S-domains in HS to explore a variety of orientations as they combine with different proteins. It has been suggested that flexibility of the $\mathrm{N}$-acetylated regions assists the simultaneous interaction of two S-domains with dimeric proteins such as IFN- $\gamma(22)$, TGF- $\beta$ (23), and IL-8 (24), which have separate binding sites on each subunit. Concerted bending of these $\mathrm{N}$-acetyl-rich "spacer" regions has been proposed to explain how two spaced S-domains in HS interact with the platelet factor 4 tetramer (25), where the GAG recognition sites are positioned on opposite faces of the proteins.

Thus, the overall characteristics of HS are consistent with a highly accommodating polymer structure that is able to adopt various binding configurations on the large and small scale to meet the needs of protein recognition. This combination of properties may to some extent overcome the requirement for defined saccharide sequences to mediate all protein-HS interactions.

\section{Sequence specificity and shared binding sites}

HS promotes the activities of a very wide range of growth factors and cytokines, and it employs various mechanisms to exert its regulatory effects. It has been known for many years that proteins become more resistant to proteolysis and thermal denaturation when bound to HS or heparin and that HS can restore activity to VEGF that has been damaged by exposure to free radicals. Interestingly, although IFN- $\gamma$ is stabilized when bound to HS, the complex is inert, and IFN- $\gamma$ must dissociate from HS to engage its signaling receptor, because of the partial overlap of the GAGand receptor-binding domains in the protein (26).

The most notable example of sequence-specific binding of any GAG is that of the antithrombin-binding pentasaccharide, which contains a 3-O-sulfate group on a central $\mathrm{GlcNSO}_{3}$ residue $(27,28)$. In other instances the arguments for strict sequence specificity are less compelling, even for the FGFs, which have been extensively studied over the past few years (for review, see ref. 29). The HS-derived saccharides that activate FGF1 (acidic FGF) and FGF2 (basic FGF) are typical extended S-domains that contain an internal repeat sequence of IdoA,2S-GlcNSO 3 units (usually 3-5) with variable 6-O-sulfation. Although FGF1 and FGF2 are differentially responsive to sequences that vary in number and position of 6-O sulfates, there is considerable overlap in their activation profiles across a range of 
variant $\mathrm{S}$-domains $(30,31)$. Differences in patterning of S-domains by 6-O-sulfation could bias cellular responses to these FGFs (32), but it is improbable that an exclusive interaction could be achieved in this way. Greater selectivity may be possible if HS acts as a template for bringing FGF and its signaling receptors into close proximity, in which case receptor and ligand recognition domains would need to be combined in a single sequence (33). It is interesting that the minimal binding sequences for FGF1 and FGF2 (octa- and hexasaccharides, respectively) are shorter than the S-domains required for growth factor activation, and a recent significant paper has reported that a three-sugar motif of IdoaA2S-GlcNSO3,6S-IdoA,2S within an eight-sugar $\mathrm{N}$-sulfated sequence creates a highly complementary binding sequence for FGF1 (34). Sulfation at C-6 is not essential for binding to FGF2, despite its importance for FGF2 activation.

There are twenty known members of the FGF family, and many isoforms of their high-affinity receptors (FGFRs) are produced by differential splicing of the four FGFR genes (35). It will be interesting to see if naturally occurring sequences can be identified that are specifically tuned to particular FGF/FGFR combinations.

\section{Lessons from developmental genetics}

Genetic experiments in Drosophila and mice have provided compelling evidence that the assembly of a normal HS structure is essential for complete development of animal embryos $(2,36)$. In Drosophila, genetic screens have identified mutations in HS biosynthetic enzymes that reproduce some of the phenotypic traits of mutations in the genes of developmentally regulated morphogens, such as the wingless and hedgehog proteins. HS seems to play a key role in the transfer of wingless to its multi-span receptor, frizzled (37). In the case of the hedgehog protein, HS dictates the spatial distribution of this morphogen, which acts across fields of cells responsive to different concentrations of the inducer $(36,38)$.

Studies with mice homozygous for mutations in HS biosynthetic enzymes have also been very revealing, particularly in the context of potential adaptive mechanisms employed by HS (see Forsberg and Kjellén, this Perspective series, ref. 39). Embryos deficient in one of the $\mathrm{N}$-deacetylase/N-sulphotransferase enzymes (NDST-1) synthesize an aberrant polysaccharide with short S-domains and low overall sulfation, whereas an HS species lacking 2-O-sulfate groups is synthesized by embryos with mutations in the single HS-2OST gene (C. Merry et al., unpublished observation). These mutant embryos are not normal, and they die in the early neonatal period. The $\mathrm{NDST1}^{-/-}$mice have impaired lung development and die as a result of respiratory failure. Loss of function of the gene for HS-2OST leads to bilateral renal agenesis, together with milder phenotypic changes in the skeleton and the nervous system (40). However, many developmental processes appear to occur normally in these knockout mice, suggesting that the altered polysaccharides that these embryos synthesize are able to support many of the activities of the wild-type HS. It could be argued that the atypical HS structures are nonfunctional and compensated for by HS-independent signaling pathways. In this regard, the recent report on the effects of disruption of one of the two HS-polymerases (the Ext genes) is very significant (41). The $E x t 1^{-/-}$mice are largely deficient in HS and die at the gastrula stage, indicating an absolute requirement for HS for progressive embryonic growth and morphogenesis. It follows that the aberrant HS species synthesized by the $\mathrm{NDST1}^{-/-}$and $\mathrm{HS}-2 \mathrm{OST^{-/ }}$ embryos must support some of the activities of the wild-type polysaccharide to enable these embryos to survive until birth. It thus appears that deficiencies in polymer sulfation can be partly overcome by compensatory mechanisms inherent in the basic physical properties of the HS chain. However, the phenotypic abnormalities of the mutant embryos indicate that certain critical stages of development require specific HS sequences.

\section{Reduced dimensionality and catalytic effects}

HSPGs are abundant components on plasma membranes, and it is likely that the long, flexible, polyanionic HS chains will be very effective in capturing and concentrating growth factors and restricting their diffusion to the quasi-two-dimensional network of polysaccharides around the cell surface $(42,43)$. This will enhance the probability of signal transduction by increasing the frequency of collisions between ligands and their highaffinity receptors (i.e., a catalytic effect). The requirements for specific growth factor recognition sequences in the context of the approximating effects of cell surface HS on ligand-receptor interactions are unclear. If this phenomenon is of general importance it is likely to be more efficiently realized by HS chains that can accommodate a wide range of growth factors able to share common binding domains.

The high ligand concentrations that can, in theory, be achieved by their interaction with cell surface HS do not fully explain all of the actions of the polysaccharide in promoting cell growth. Cultured cells deficient in cell surface HS usually fail to proliferate in the presence of HS-dependent growth factors even if growth factor concentrations are significantly increased $(44,45)$, so the HS must be doing something more than increasing the probability of ligand-receptor binding. HS could enhance the affinity of ligand-receptor interactions by inducing an activating conformational change in the ligand, but no convincing evidence for this has been published to date. FGF2 binds to FGFRs expressed on HS-depleted cells and elicits downstream signals, but these are short-lived in comparison with HS-mediated signaling (46). This suggests that one of the functions of HS is to stabilize the ligand-receptor complex, perhaps by a cross-bridging mechanism.

\section{Dimerization models and crystal structures}

Several groups have independently proposed that the activation of monomeric growth factors, such as the FGFs and HGF, occurs as a result of their association as dimers or oligomers on the HS chain. The rationale for this is twofold: higher-order structures are assumed to favor receptor dimerization, which is essential for 
transmembrane signaling, and, in the case of the FGFs, active site HS sequences are 10-14 sugars in length, making them long enough to accommodate two growth factors $(6,44,47)$.

A number of HS-FGF dimer arrangements have been described with the monomer units in cis or trans orientation, the former with direct protein-protein contacts at the dimer interface (see, for example, refs. 48, 49). Although the arguments for HS-assisted formation of ligand dimers are plausible, with good experimental support, other possibilities should be considered. Molecular modeling and protein structure prediction indicate that monomeric FGF2 could dimerize FGFRs by associating with two receptor-binding regions on the protein surface (50). In this model, FGF2 displayed unassisted binding to one of the dimerized FGFRs, but the interaction with the second receptor was stabilized by HS. Moreover, in GAG-protein cross-linking experiments it was found that 1:1 covalent complexes were formed between FGF2 and S-domains isolated from HS. These complexes were biologically active, suggesting that the minimum mitogenic unit is a single FGF2 bound to a saccharide activator (51).

The proposition that monomeric FGF complexed to $\mathrm{HS}$ is the basic active unit is supported by crystal structures of signaling complexes of heparin and FGF1/FGFR2, which revealed that ligand and receptor formed a 2:2 tetrameric assembly with each of the FGF1/FGFR2 dimers in association with separate heparin decasaccharides. The saccharides in these structures are arranged in an antiparallel fashion and make extensive contacts with a canyon of positive charge that extends across the ligand receptor pairs (52).

However, a contrasting view of the role of heparin was deduced from crystal structures of FGF2 and FGFR1. Here again, growth factor and receptor are seen to form a 2:2 tetramer but only a single asymmetric heparin decasaccharide is present in the complex (21), and the saccharide binds two noncontacted FGF1 molecules in an offset, trans arrangement that tethers two FGFRs into the assembly. The saccharide interacts with only one of these receptors and induces a modification in receptor structure that was predicted to be essential for an active signaling complex to be formed. Thus, in this study and in the work on the FGF2/FGFR1 complexes, heparin - and by inference HS - is featured as an integral component of the signaling assembly. This argues against an exclusively catalytic mechanism for polysaccharide-mediated transfer of ligands to receptors.

In the future, it will be important to compare the crystal structures of putative signalling assemblies formed under similar experimental conditions (different procedures were used in the above studies) and in the presence of HS-derived saccharides of near-homogenous structure. It will be especially interesting to see whether bioactive HS sequences of relatively low sulfation engage ligands and receptors in the same way as highly sulfated heparin. Our present state of knowledge indicates that the complex and multifaceted FGF/FGFR receptor system demands a flexible role for the HS coreceptor, and there may be more than one solution to the problem of how to form signaling complexes.

\section{Conclusions}

It is proposed that HS executes many of its actions by means of a limited range of sequences, despite the immense potential for sequence diversity inherent in its disaccharide unit structures (20). For example, over $1,000,000$ different sequences could be represented in an HS-octasaccharide (20), a figure which vastly exceeds the number of genes in the human genome. The physical properties of the HS chains are predicted to be a key factor in limiting the requirement for unique sequences for each HS-protein interaction. An a la carte menu of sequences, based on the predominant S-domain format of IdoA,2S-GlcNSO ${ }_{3}$ repeat units with variable 6-O-sulfation, may be compatible with the regulation of the majority of proteins that interact with HS. Cell-type variations in HS structure $(53,54)$ may well be due in large part to the synthesis of different proportions of the standard menu. However, such sequences will not satisfy proteins with more discerning tastes; these will require a special diet flavored with rare ingredients such as 3-O-sulfation and $\mathrm{N}$-unsubstituted glucosamine (55). At the interface of proteomics and glycoinformatics, sequence analysis of naturally occurring HS and heparin saccharides and their chemically modified derivatives (for example, selectively desulfated structures) will be essential for determining the critical functional groups involved in the recognition and activation of different proteins. The recent rapid advances in organic synthesis of HS and heparin saccharides will undoubtedly be helpful in this regard (56). Eventually, minimal binding sequences with improved selectivity of action will be identified. Many of these minimal sequences may not occur naturally, because constraints imposed by the specificities of the HS-polymer modifying enzymes will restrict the degree of sequence diversity in the HS chain. Information on minimal binding and activating sequences will be vital for understanding the rules of engagement in HS-protein recognition and the biological consequences that follow when such interactions occur. Moreover, new insights should emerge for the design of saccharide mimetics that can specifically target individual growth factors, chemokines, and other proteins involved in human disease.

1. Kjellen, L., and Lindhal, U. 1991. Proteoglycans: structures and interactions. Annu. Rev. Biochem. 60:443-475.

2. Perrimon, N., and Bernfield, M. 2000. Specificities of heparan sulphate proteoglycans in developmental processes. Nature. 404:725-728.

3. Lyon, M., and Gallagher, J.T. 1998. Bio-specific sequences and domains in heparan sulphate and the regulation of cell growth and adhesion. Matrix Biol. 17:485-493.

4. Couchman, J.R., and Woods, A. 2000. Signalling through the syndecan proteoglycans. In Proteoglycans: structure, biology and molecular interactions. R.V. Iozzo, editor. Marcel Dekker Inc. New York, New York, USA. 147-160.

5. Iozzo, R.V. 1998. Matrix proteoglycans: from molecular design to cellular function. Annu. Rev. Biochem. 67:609-652.

6. Gallagher, J.T., and Lyon, M. 2000. Molecular structure of heparan sulfate and interactions with growth factors and morphogens. In Proteoglycans: structure, biology and molecular interactions. R.V. Iozzo, editor. Marcel Dekker Inc. New York, New York, USA. 27-59.

7. Iozzo, R.V. 2001. Heparan sulfate proteoglycans: intricate molecules with intriguing functions. J. Clin. Invest. 108:349-355.

8. Zhang, L., and Esko, J.D. 1994. Amino acid determinants that drive heparan sulfate assembly in a proteoglycan. J. Biol. Chem. 269:19295-19299.

9. Esko, J.D., and Lindahl, U. 2001. Molecular diversity of heparan sulfate. J. Clin. Invest. 108:169-173 
10. Turnbull, J.E., and Gallagher, J.T. 1991. Distribution of iduronate-2-sulphate in heparan sulphate. Evidence for an ordered polymeric structure. Biochem. J. 273:553-559.

11. Lyon, M., Deakin, J.A., and Gallagher, J.T. 1994. Liver heparan sulfate structure. A novel molecular design. J. Biol. Chem. 269:11208-11215.

12. Maccarana, M., Sakura, Y., Tawada, A., Yoshida, K., and Lindahl, U. 1996. Domain structure of heparan sulfates from bovine organs. J. Biol. Chem. 271:17804-17810.

13. Merry, C.L., Lyon, M., Deakin, J.A., Hopwood, J.J., and Gallagher, J.T. 1999. Highly sensitive sequencing of the sulfated domains of heparan sulfate. J. Biol. Chem. 274:18455-18462.

14. Safaiyan, F., Lindahl, U., and Salmivirta, M. 2000 Structural diversity of $\mathrm{N}$-sulphated heparan sulphate domains. Biochemistry. 39:10823-10830.

15. Liu, J., et al. 1999. Heparan sulfate D-glucosaminyl 3-O-sulfotransferase$3 \mathrm{~A}$ sulfates $\mathrm{N}$-unsubstituted glucosamine residues. J. Biol. Chem. 274:38155-38162

16. Van den Born, J., et al. 1995. Presence of N-unsubstituted glucosamine units in native heparan sulfate revealed by a monoclonal antibody. J. Biol. Chem. 270: 31303-31309.

17. Mulloy, B., Forster, M.J., Jones, C., and Davies, D.B. 1993. N.m.r. and molecular-modelling studies of the solution conformation of heparin. Biochem. J. 293:849-858.

18. Mulloy, B., and Forster, M.J. 2000. Conformation and dynamics of heparin and heparan sulfate. Glycobiology. 10:1147-1156.

19. Faham, S., Hileman, R.E., Fromm, J.R., Linhardt, R.J. and Rees, D.C 1996. Heparin structure and interactions with basic fibroblast growth factor. Science. 271:1116-1120.

20. Sasisekharan, R., and Venkataraman, G. 2000. Heparin and heparan sulfate: biosynthesis, structure and function. Curr. Opin. Chem. Biol. 4:626-631.

21. Pellegrini, L., Burke, D.F., von Delft, F., Mulloy, B., and Blundell, T.L. 2000. Crystal structure of fibroblast growth factor receptor ectodomain bound to ligand and heparin. Nature. 407:1029-1034.

22. Lortat-Jacob, H., Turnbull, J.E., and Grimaud, J.A. 1995. Molecular organization of the interferon $\gamma$-binding domain in heparan sulphate. Biochem. J. 310:497-505.

23. Lyon, M., Rushton, G.R., and Gallagher, J.T. 1997. The interaction of transforming growth factor- $\beta$ with heparin/heparan sulfate is isoformspecific. J. Biol. Chem. 272:18000-18006.

24. Spillmann, D., Witt, D., and Lindahl, U. 1998. Defining the Interleukin8-binding domain of heparan sulfate. J. Biol. Chem. 273:15487-15493.

25. Stringer, S.E., and Gallagher, J.T. 1997. Specific binding of the chemokine platelet factor 4 to heparan sulfate. J. Biol. Chem. 272:20508-20514.

26. Sadir, R., Forest, E., and Lortat-Jacob, H. 1998. The heparan sulfate binding sequence of interferon- $\gamma$ increased the on rate of the interferon- $\gamma$ interferon- $\gamma$ receptor complex formation. J. Biol. Chem. 273:10919-10925.

27. Spillmann, D., and Lindahl, U. 1994. Glycosaminoglycan-protein interactions: a question of specificity. Curr. Opin. Struct. Biol. 4:677-682.

28. Jin, L., et al. 1997. The anticoagulant activation of antithrombin by heparin. Proc. Natl. Acad. Sci. USA. 94:14683-14688.

29. Gallagher, J.T. 1998. The interaction and regulation of basic and acidic fibroblast growth factors by heparan sulfate. Trends Glycosci. Glycobiol. 10:137-144.

30. Pye, D.A., Vives, R., Turnbull, S.E., Hyde, P., and Gallagher, J.T. 1998. Heparan sulfate oligosaccharides require 6-O-sulfation for promotion of basic fibroblast growth factor mitogenic activity. J. Biol. Chem. 273:22936-22942.

31. Pye, D.A., Vives, R.R., Hyde, P., and Gallagher, J.T. 2000. Regulation of FGF-1 mitogenic activity by heparan sulfate oligosaccharides is dependent on specific structural features: differential requirements for the modulation of FGF-1 and FGF-2. Glycobiology. 10:1183-1192.

32. Brickman, V.G., et al. 1998. Structural modification of fibroblast growth factor binding heparan sulfate at a determinative stage of neural development. J. Biol. Chem. 273:4350-4359.

33. Guimond, S.E., and Turnbull, J.E. 1999. Fibroblast growth factor receptor signalling is dictated by specific heparan sulfate saccharides. Curr. Biol. 9:1343-1346.
34. Kreuger, J., Salmivirta, M., Sturiale, L., Gimenez-Gallego, G., and Lindahl, U. 2001. Sequence analysis of heparan sulphate epitopes with graded affinities for FGF1 and FGF2. J. Biol. Chem. In press.

35. Coutts, J.C., and Gallagher, J.T. 1995. Receptors for fibroblast growth factors. Immunol. Cell Biol. 73:584-589.

36. Lander, A.D., and Selleck, S.B. 2000. The elusive functions of proteoglycans: in vivo veritas. J. Cell Biol. 148:227-232.

37. Tsuda, M., et al. 1999. The cell-surface proteoglycan Dally regulates Wingless signalling in Drosophila. Nature. 400:276-280.

38. Bellaichie, Y., The, I., and Perrimon, N. 1998. Tout-velu is a Drosophila homologue of the putative tumour suppressor Ext-1 and is needed for Hh diffusion. Nature. 394:85-88.

39. Forsberg, E., and Kjellén, L. 2001. Heparan sulfate: lessons from knockout mice. J. Clin. Invest. 108:175-180.

40. Bullock, S.L., Fletcher, J.M., Beddington, R.S., and Wilson, V.A. 1998 Renal agenesis in mice homozygous for a gene trap mutation in the gene encoding heparan sulfate 2-sulfotransferase. Genes Dev. 12:1894-1906.

41. Lin, X., et al. 2000. Disruption of gastrulation and heparan sulfate biosynthesis in EXT1-deficient mice. Dev. Biol. 224:299-311.

42. Lander, A.D. 1998. Proteoglycans: master regulators of molecular encounter? Matrix Biol. 17:465-472.

43. Schlessinger, J., Lax, I., and Lemmon, M. 1995. Regulation of growth factor activation by proteoglycans: what is the role of the low affinity receptors? Cell. 83:357-360.

44. Walker, A., Turnbull, J.E., and Gallagher, J.T. 1994. Specific heparan sulfate saccharides mediate the activity of basic fibroblast growth factor. $J$. Biol. Chem. 269:931-935.

45. Fannon, M., and Nugent, M.A. 1996. Basic fibroblast growth factor binds its receptors, is internalized, and stimulates DNA synthesis in Balb/c3T3 cells in the absence of heparan sulfate. J. Biol. Chem. 271:17949-17956.

46. Delehedde, M., et al. 2000. Fibroblast growth factor-2 stimulation of p42/44MAPK phosphorylation and IkB degradation is regulated by heparan sulfate/heparin in rat mammary fibroblasts. J. Biol. Chem. 275:33905-33910.

47. Maccarana, M., Casu, B., and Lindahl, U. 1993. Minimal sequence in heparin/heparan sulfate required for binding of basic fibroblast growth factor. J. Biol. Chem. 268:23898-23905.

48. Venkataraman, G., Shriver, Z., Davis, J.C., and Sasisekharan, R. 1999. Fibroblast growth factors 1 and 2 are distinct in oligomerization in the presence of heparin-like glycosaminoglycans. Proc. Natl. Acad. Sci. USA. 96:1892-1897.

49. Chirgadze, D.Y., et al. 1999. Crystal structure of the NK1 fragment of HGF/SF suggests a novel mode for growth factor dimerization and receptor binding. Nat. Struct. Biol. 6:72-79.

50. Springer, B.A., et al. 1994. Identification and concerted function of two receptor binding surfaces on basic fibroblast growth factor is required for mitogenesis. J. Biol. Chem. 269:26879-26884.

51. Pye, D.A., and Gallagher, J.T. 1999. Monomer complexes of basic fibroblast growth factor and heparan sulfate oligosaccharides are the minimal functional unit for cell activation. J. Biol. Chem. 274:13456-13461.

52. Schlessinger, J., et al. 2000. Crystal structure of a ternary FGF-FGFRheparin complex reveals a dual role for heparin in FGFR binding and dimerization. Mol. Cell. 6:743-750.

53. Gallagher, J.T., and Walker, A. 1985. Molecular distinctions between heparan sulphate and heparin. Analysis of sulphation patterns indicates that heparan sulfate and heparin are separate families of $\mathrm{N}$-sulphated polysaccharides. Biochem. J. 230:665-674.

54. Van Kuppevelt, T.H., Dennissen, M.A., van Venrooij, W.J., Hoet, R.M., and Veerkamp, J.H. 1998. Generation and application of type-specific anti-heparan sulfate antibodies using phage display technology. Further evidence for heparan sulfate heterogeneity in the kidney. J. Biol. Chem. 273:12960-12966.

55. Shukla, D., et al. 1999. A novel role for 3-O-sulfated heparan sulfate in herpes simplex virus 1 entry. Cell. 99:13-22.

56. Obadiah, J., Plante, J., Palmacci, E.R., and Seeberger, P.H. 2001. Automated solid-phase synthesis of oligosaccharides. Science. 291:1523-1527. 